\title{
Injury Mechanism of Sub-Micron Calcium Oxalate Monohydrate and Dihydrate Crystals on Renal Epithelial Cells
} Poonam BHADJA, Kai YU, Xiu-qun YAO and Jian-ming OUYANG

Department of Chemistry, Jinan University, Guangzhou, 510632, China

${ }^{\dagger} E$-mail: toyjm@jnu.edu.cn

Keywords: Submicron Crystal, Concentration Effect, Cell Injury, Crystal Adhesion.

\begin{abstract}
Objective: This study aims to compare the cytotoxicity and adhesion of submicron calcium oxalate monohydrate (COM) and dehydrate (COD) crystals toward African green monkey renal epithelial cells (Vero) to reveal the mechanism of kidney stone formation at cellular level. Methods: The injury effect of COM and COD crystals in Vero was investigated by detecting the change of cell viability, lactate dehydrogenase (LDH) content and hyaluronan (HA) expression amount. The adhesion of crystals on cell surface was observed using scanning electron microscope; and the crystal adhesion amount was measured using inductively coupled plasma emission spectrometer. Results: The cellular injury induced by crystals was in a dose-dependent manner in the concentration range of $100 \sim 400 \mu \mathrm{g} / \mathrm{mL}$; COM caused more serious injury than COD, the adhesion amount of COM on Vero was significantly greater than COD. Conclusions: The damage of submicron COM is larger than submicron $\mathrm{COD}$, the adhered COM crystals are more easily aggregated. The results in this paper indicated that the presence of high concentration of COM crystals in urine is more likely to increase the risk of stone formation than COD crystals.
\end{abstract}

\section{Introduction}

The main component of kidney stone is calcium oxalate monohydrate (COM) and its incidence is about twice of calcium oxalate dehydrate (COD) [1], attributing to the adhesion ability of COM on renal epithelial cell is stronger than COD crystal. COD is thermodynamically unstable and can be slowly transformed to stable COM crystals in urine. Wesson et al [2] confirmed that the adhered amount of COM crystals on renal tubular cell is $50 \%$ higher than COD.

The study in vivo confirmed that it needs 5-10 min for the formed urine crystals to pass through kidney. The inner diameter of the pipeline is about 15-60 $\mu \mathrm{m}$. Since the crystal growth rate in urine is about $1-2 \mu \mathrm{m} / \mathrm{min}$, therefore, crystal can not grow to large enough to block the urine pipeline within 5-10 min, these crystals will be washed away by urine if no crystal adhesion occurs.

In addition to crystal nucleation, growth and aggregation, the crucial mechanism for calcium oxalate kidney stone formation is the adhesion of crystals on renal tubular epithelial cells [3]. Only the crystals were adhered to the renal epithelial cells, can they have enough time to grow to larger size on cell surface, and eventually lead to the formation of kidney stones. That is, non-adhered crystals have less risk to kidney stone formation.

Hence, we studied the adhesion difference of submicron COM and COD crystals on Vero cells in this work, and focused on the concentration effect of crystals, in order to further understand the molecular and cellular mechanism of kidney stone formation. 


\section{Materials and Methods}

\section{Materials and Apparatus}

African green monkey renal epithelial (Vero) cells were purchased from Shanghai Cell Bank. Cell proliferation assay kit (CCK-8) and Lactate dehydrogenase (LDH) kit were purchased from Dojindo Laboratories (Kumamoto, Japan). All conventional reagents used were analytically pure.

X-L type environmental scanning electron microscope (SEM, Phillps, Eindhoven, Netherlands), laser confocal microscope (LSM510 META DUO SCAN, ZEISS, Germany), enzyme mark instrument (Safire2 ${ }^{\mathrm{TM}}$, Tecan, Männedorf, Switzerland).

\section{Preparation and Characterization of Submicron Calcium Oxalate Monohydrate (COM) and Dehydrate (COD) Crystals}

Sub-micron COM and COD crystals were prepared according the previous method [4], XRD and FT-IR showed that they were our target product.

\section{Cell Culture, Cell Viability Assay, Lactate Dehydrogenase (LDH) Release Assay, and Cell Death Assay}

These experiments were carried out according to reference [4].

\section{HA Expression Assay}

According to reference [4], after synchronization, the cells were divided as control group and crystal groups. One hundred microliters of FITC-avidin (fluorescein isothiocyanate-avidin) was added to the cells and incubated for $1 \mathrm{~h}$. The cells were washed with PBS three times for 5 min each. DAPI staining solution was then added to the cells and incubated for $4 \mathrm{~min}$. Finally, the prepared samples were mounted with anti-fade fluorescence mounting medium and observed in a confocal microscope. HA expression and cell nuclei were stained green and blue, respectively.

\section{SEM Observation and Crystal Adhesion Amount Measurement}

After crystals exposed to cells for $6 \mathrm{~h}$, the supernatant was removed by suction, the cell-crystal mixture fixed in PBS $2.5 \%$ glutaraldehyde at $4{ }^{\circ} \mathrm{C}$ for $24 \mathrm{~h}$, fixed with $1 \%$ $\mathrm{OsO}_{4}$, dehydrated in gradient ethanol $(30 \%, 50 \%, 70 \%, 90 \%$ and $100 \%$, respectively), eventually fixed with isoamyl acetate, dried under the critical point of $\mathrm{CO}_{2}$, and treated with gold sputtering, finally the cell-crystal mixture was observed under SEM. The amount of COM or COD crystals adhered on cells was measured using ICP method and the results were expressed in $\mu \mathrm{g} / \mathrm{cm}^{2}$.

\section{Results}

\section{Preparation and Characterization of COM and COD Crystals}

Figure 1 showed the SEM images of submicron COM and COD crystals, their average particle size was $200 \pm 40 \mathrm{~nm}$ and $200 \pm 60 \mathrm{~nm}$, respectively.

\section{Cell Viability Change After Crystal Adhesion}

The effect of submicron COM and COD on the viability of Vero cells was shown in Figure 2a. Both of them can decrease the cell viability, indicating that they are toxic to Vero cells. The cytotoxicity increased with the increase of crystal concentration. That is, COM and COD crystals presented concentration-dependent toxicity in Vero. 
At the same crystal concentration, the cell viability of COM group was always smaller than COD group, indicating that submicron COM has significantly larger cytotoxicity than COD.

\section{Lactate Dehydrogenase (LDH) Release Change After Crystal Adhesion}

LDH is a glycolytic enzyme which exists in all tissues and cells, especially with a higher level in kidney. Once the cell membrane was ruptured, LDH is released out of cells [5]. The more the LDH releases, the greater means the cell injury degree. Thus, LDH release amount was considered as a marker of cell membrane integrity loss and cell damage. Compared with the normal control group (no crystal), the LDH release of COM group and COD group was increased (Figure 2b), and LDH release gradually increased with the increase of crystal concentration. The increase of LDH release caused by COM crystals is always higher than COD crystals, which suggests that submicron COM has stronger ability to destroy the cell membrane than submicron COD.

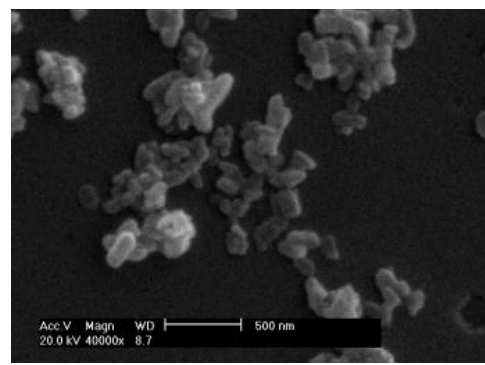

(a)

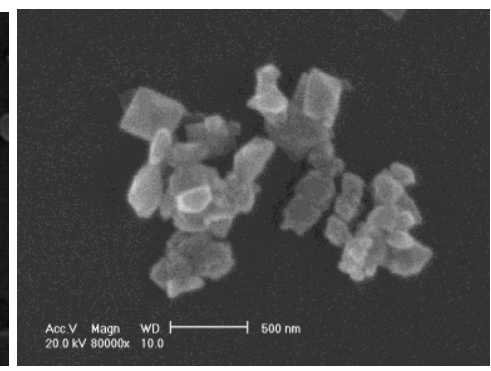

(b)

Figure 1. SEM images of submicron COM (a) and COD (b). The bars: $500 \mathrm{~nm}$
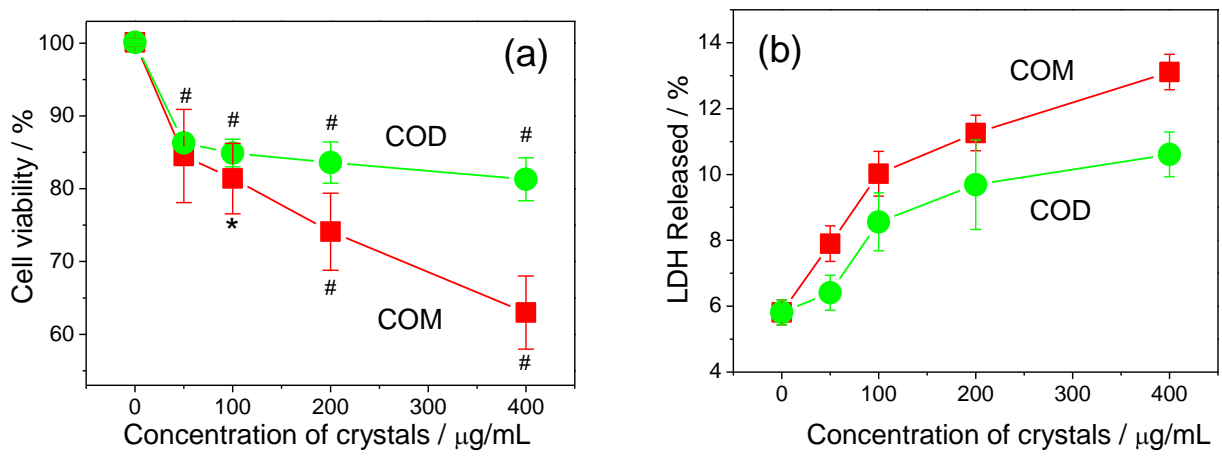

Figure 2. Change in cell viability (a) and LDH release amount (b) of Vero cells after exposure to submicron COM and COD for $6 \mathrm{~h}$.

\section{Propidium Iodide (PI) Staining After Crystal Adhesion}

PI can not penetrate through normal cell membranes but can pass through necrotic cell membranes and bind to DNA in the nucleus and emit red fluorescence. The number of red-stained cells in the control group was low (Figure 3A). The number of red-stained cells and fluorescence intensity in COM treatment groups were greater than those in COD groups at the same crystal concentration (Figure 3B). With the increase of crystal concentration, the relative fluorescence intensity becomes more and more stronger. Figure 3 suggested that COM and COD crystals could simultaneously cause apoptosis and necrosis, but COM caused more cell death than COD crystals. 


\section{Hyaluronan (HA) Expression After Crystal Adhesion}

HA expression quality change on Vero cell surface after adhesion of different concentrations of submicron COM and COD was shown in Figure 4. After adhesion with COM, green fluorescence on Vero surface enhanced with the increase of crystal concentration (Figure 4A), which suggested that the expression quantity of HA increased gradually. When the concentration of COM is $400 \mu \mathrm{g} / \mathrm{mL}$, the relative fluorescence intensity is $736 \pm 40$ (Figure 4B), which is significantly higher than the concentration of $100 \mu \mathrm{g} / \mathrm{mL}(227 \pm 11)$ and no crystal adhesion (122 \pm 8$)$ respectively. At the same crystal concentration, green fluorescence of COD group is weaker than COM group.
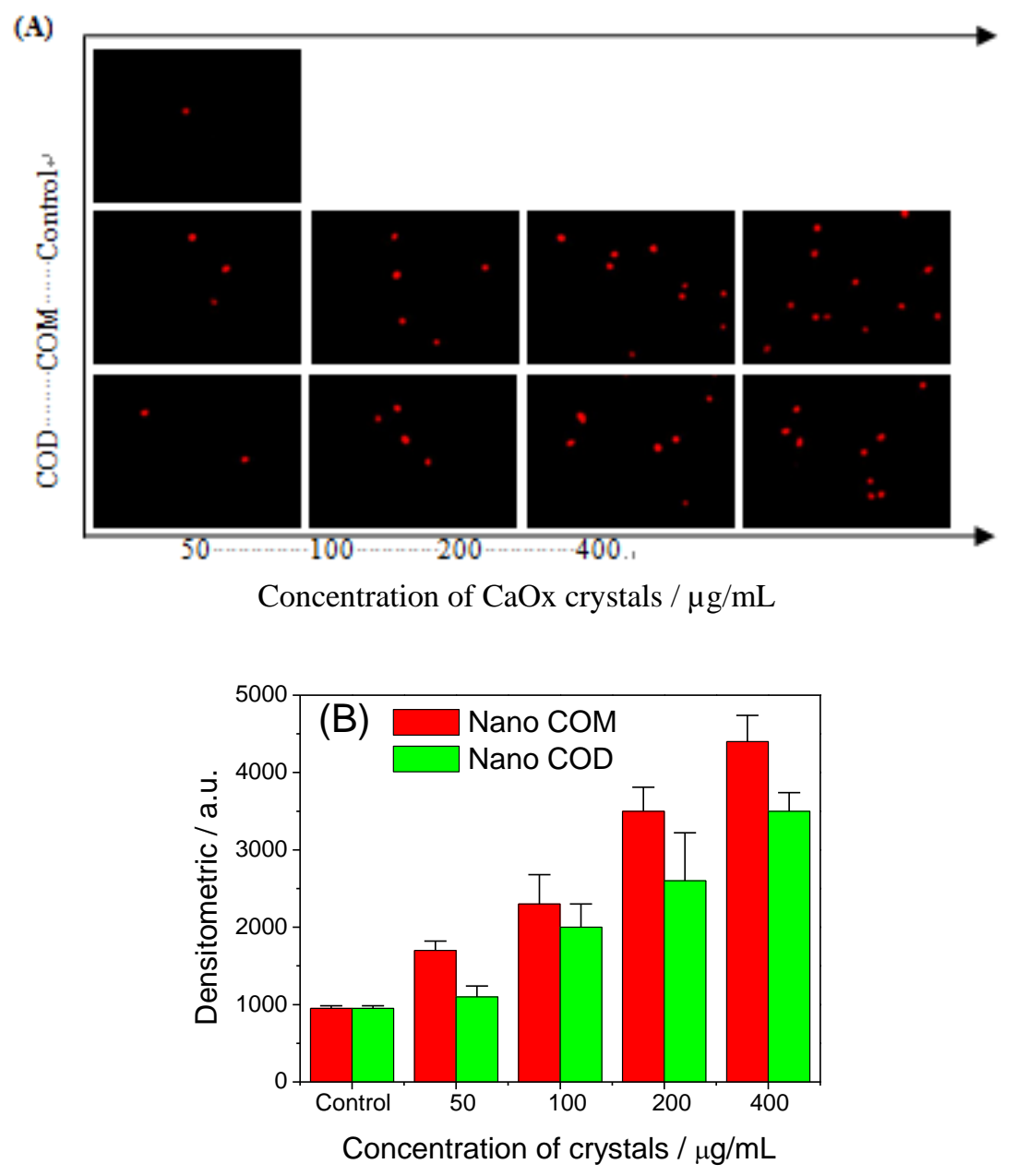

Figure 3. PI staining assay of Vero cells after exposure to different concentrations of COM and COD crystals for 6 h. (A) Fluorescence microscope images (300x) of dead cells. (B) Quantitative results of dead cells.

\section{SEM Observation of Crystal Adhered on Vero}

The SEM images of Vero cells after exposure to submicron COM and COD for $6 \mathrm{~h}$ were shown in Figure 5. With the concentration of crystals increases, the number of the adhered crystals and the size of aggregated crystals increased. By comparison, submicron COD aggregated only at high concentration (Figure. 6f). Also, the number of submicron COD adhered on the Vero cells was less than COM at the same crystal concentration.

The adhesion amount of different concentration of COM and COD adhered on 
Vero was detected using ICP (Figure 6). With increasing concentrations of crystals, the amount of crystal adherence increased constantly, and the adhesion amount of COD was lower than COM crystals.

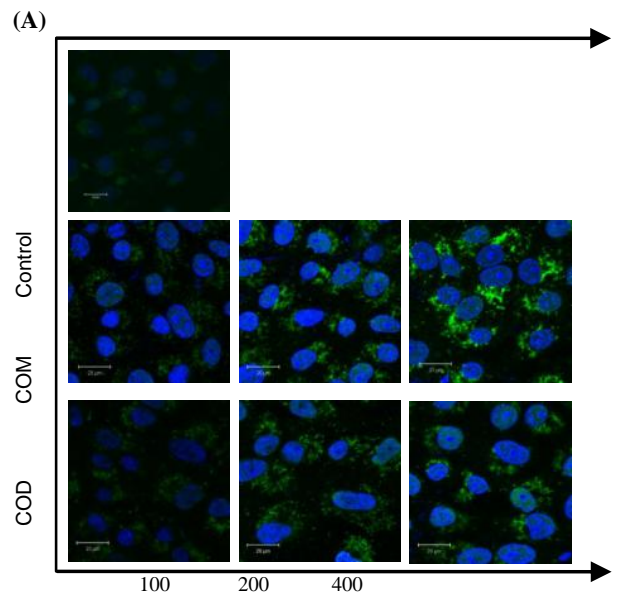

Concentration of $\mathrm{CaOx}$ crystals / $\mu \mathrm{g} / \mathrm{mL}$

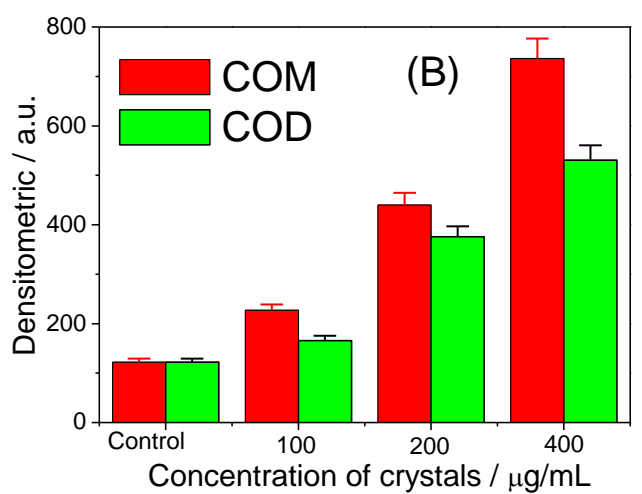

Figure 4. Hyaluronan expression on Vero cells after exposure to different concentrations submicron $\mathrm{COM}$ and COD for $6 \mathrm{~h}$. (A) Laser scanning confocal microscope images; (B) relative fluorescence intensity. The nucleus is blue and HA is green. Scale bars: $20 \mu \mathrm{m}$. 


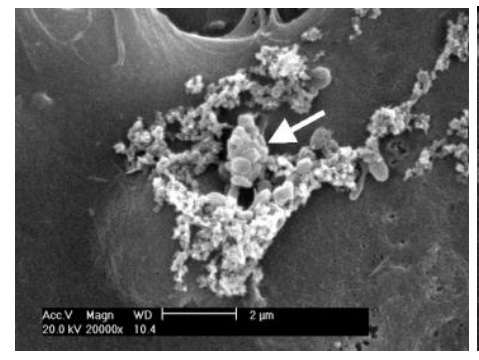

(a)

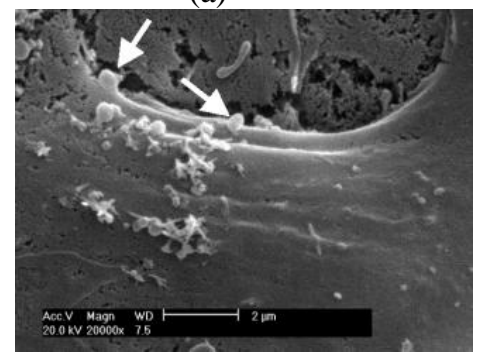

(d)

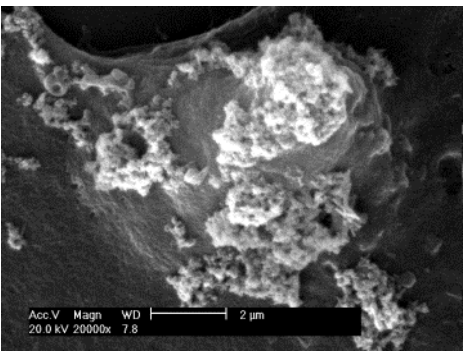

(b)

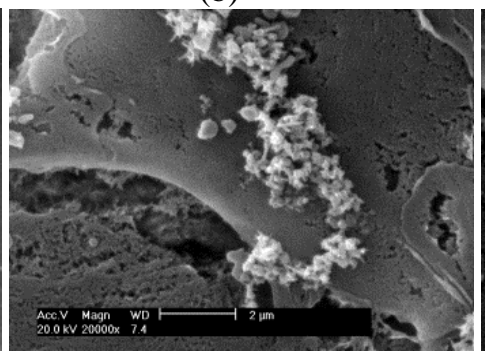

(e)

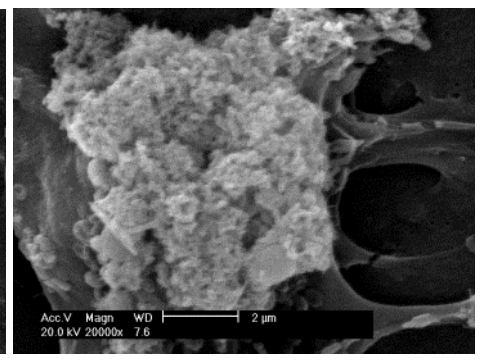

(c)

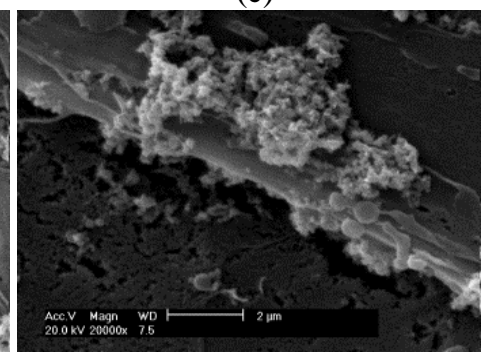

(f)

Figure 5. SEM images of Vero cells after exposure to different concentration of submicron COM and COD. (a,d) 100; (b,e) 200; (c,f) $400 \mu \mathrm{g} / \mathrm{mL}$. (a-c) COM; (d-f) COD.

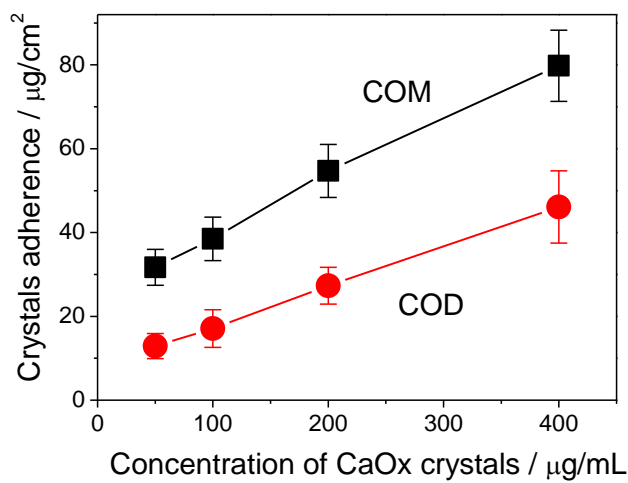

Figure 6. Adhesion amount of COM and COD crystals on Vero cells detected using ICP. Adhesion time: $6 \mathrm{~h}$.

\section{Conclusions}

Submicron COM and COD can cause the decrease of cell viability and increase of LDH release, and also increase cell mortality, it suggested that both COM and COD have cytotoxicity and injury effect to Vero cells. After injured, the HA expression amount, crystal adhesive amount and the aggregated crystals showed concentrationdependent effect in the concentration range of 50 to $400 \mu \mathrm{g} / \mathrm{mL}$. Submicron COM has significantly larger cytotoxicity than COD. Therefore, the increased concentration of COM significantly increased the cell injury.

\section{Acknowledgments}

This work is supported by the Natural Science Foundation of China (NO. 21371077). 


\section{References}

[1] M.S. Ansari, N.P. Gupta, A.K. Hemal, P.N. Dogra, A. Seth, M. pectrum of stone composition: structural analysis of 1050 upper urinary tract calculi from northern India. Int. J. Urol. 12, 12 (2005).

[2] J.A. Wesson, E.M. Worcester and J.G. Kleinman. Role of anionic proteins in kidney stone formation: interaction between model anionic polypeptides and calcium oxalate crystals. J. Urol. 163, 1343 (2000).

[3] B.H. Wang, B.L. Wu, J. Liu, W.M. Yao, D. Xia, L. Li, Z.Q. Chen, Z.Q. Analysis of Altered MicroRNA Expression Profiles in Proximal Renal Tubular Cells in Response to Calcium Oxalate Monohydrate Crystal Adhesion: Implications for Kidney Stone Disease. Plos One. 9, e101306 (2014).

[4] X.Y. Sun, J.M. Ouyang, A.J. Liu, Y.M. Ding, Y.B. Li and Q.Z. Gan. Preparation, characterization, and in vitro cytotoxicity of COM and COD crystals with various sizes. Mater. Sci. Eng. C. 57, 147 (2015).

[5] X.Y. Sun, J.M. Ouyang, W.Y. Zhu, Y.B. Li and Q.Z. Gan, Size-dependent toxicity and interactions of calcium oxalate dehydrate crystal on Vero renal epithelial cells. J. Mater. Chem. B. 3, 1864 (2015). 